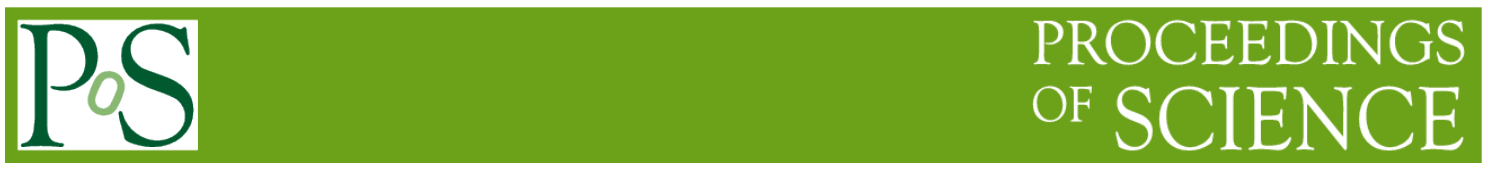

\title{
Cloud Computing Strategy Analysis in Small and Medium-Sized Enterprises
}

\author{
Liping $\mathbf{L i}^{12}$ \\ Yunnan University of Finance and Economics \\ Kunming, Yunnan, 650221, China \\ E-mail: I Iplrs@126. com
}

In order to effectively solve the resource dynamic demand and the final cost in small and medium-sized enterprises (SMEs), reduce costs, improve the management, promote the development of informationization, and realize the resource integration for SMEs, this paper proposes the cloud computing strategies for SMEs. Based on investigation, the experiment, the literature research, the experience summary and so on, this paper analyzes the competition environment of the SMEs in modern commercial society and the cloud computing value of the SMEs, and elaborates the cloud computing strategy of SMEs from three aspects: IaaS, PaaS and SaaS. This paper also explains the steps from IT to cloud computing for SMEs, the cloud computing model of SMEs, gives the basic cloud computing framework, and raises cloud computing security strategy for SMEs. Empirical results show that it is successful that SMEs' IT has been evolved to the cloud computing, which may assist the companies in reducing costs, improving management and allocating resources dynamically. As the cloud computing has a significant impact on the enterprise., the cloud computing is the key factor to form and improve the competitiveness of SMEs.

CENet2017

22-23 July 2017

Shanghai, China

\footnotetext{
${ }^{1}$ Speaker

${ }^{2}$ Correspongding Author
} 


\section{Introduction}

China has put forward the strategy of "two integrations" since the Seventeenth National Congress of the Communist Party of China, In an increasingly competitive environment and in the post crisis era, only by further management optimization, resourcesintegration, cost reduction, improvement of the IT performance (to build agile IT) can SMEs achieve the "two integrations" and take the road of innovation and development.

At present, there is no lack of researches on cloud computing both in China and foreign countries, including Architectural Requirements for Cloud Computing Systems [1], which has classified three-layered architectural requirements for cloud computing (provider requirements, enterprise requirements and user requirements); Cloud Computing Technology, Platform and Application[2], which take a global view of business model, technology, application and standard of cloud computing; and other papers include Competitive Advantage [3], Success Factors [4], Architecture and Key Technology [5], Strategy of Group Enterprise [6], Some Problems in Cloud Computing[7], Cloud Computing Security and China's Policy [8]. These previous studies have proposed so many valuable theories and methods. These early researches shall be greatly appreciated for their help in this article, but there are not so many researches for SMEs, especially in Yunnan Province of China where the development of science and technology level is not so high.

The specific contributions of this paper include: the strategies that make SMEs in Yunnan Province of China to achieve cloud computing were proposed. Our research team has established relationship with Zhi Sheng Education Technology Co., Ltd. in Yunnan Province of China, Zhi Sheng Company has been mainly engaged in cloud computing, super integration and SaaS services. Our research team has investigated the service type and service mode of Zhi Sheng, and provided cloud computing strategies for Zhi Sheng Company, including SaaS sharing and security recommendations; at the same time, out research team also learnt from Zhi Sheng Company in terms of its solution and experience of SMEs' cloud computing services in practical application in Yunnan Province of China. It has supported the integrity and practicability of the study. Finally, this paper makes summaries and experiences in the process of cloud computing of SMEs in Yunnan, China.

\section{Cloud Computing Strategy of SMEs}

\subsection{IaaS Strategies of SMEs}

The core technology of IaaS is virtual technology, the most important technological foundation of cloud computing. It has realized the logical abstraction and uniform representation of physical resources. In IaaS, resources demanded by the enterprise can be supplied and released elastically. The storage cloud enables the enterprises uncessesary to build their own disaster recovery center. They just back up the data to the cloud service provider. The server cloud can provide a virtual computing space for enterprises, and the companies can freely customize CPU, operating system, memory, hard disk, and the companies can also run applications on this virtual system; therefore, SMEs do not have to spend a lot of money in building their own IT infrastructure. On the contrary, they can enjoy profound resources in the resource pool by paying less rents to the IaaS service provider; besides, they can also put their 
virtual information center in the trust of the IaaS service provider so as to reduce the cost of technical facilities and the spending of operation and maintenance

\subsection{PaaS Strategies of SMEs}

The services of PaaS are the development, test, deployment, running of end-to-end distributed software and complex application hosting, PaaS provides services to the users through networkI. It provides users with one-stop software development services and frees the user from development, configuration and maintenance of the program environment, which is complicated and inefficient. In this sense, the users can focus on programming and the efficiency of software development can be improved. Enterprises can use the platform provided by the PaaS service provider (the programming language or tools) to develop their own applications, that is, the development of additional services as a personalized purpose to expand their core service set. For SMEs, provide them through the public cloud platforms by the organization with development ability (such as government, group, etc.). These platforms represent specific applications such as regional medical service center, regional business application platform, regional education platform, community service centers, etc. for the purpose of providing specific public services to SMEs in the region, for example, the health records management, ERP and CRM management, student management and residential property management, etc.

\subsection{SaaS Strategies of SMEs}

SaaS is a kind of service mode, which takes the network as the carrier and takes the browser as the interactive way to transmit the program or software in the server side to the remote user. If SMEs does not have the conditions to develop the required application software (such as personnel management, financial management or business management, etc.), then, SaaS service providers can build the corresponding software platform for them while being responsible for the implementation, maintenance and other affairs. SMEs just needs to lease the service provided by the cloud service providers in respect of the actual demanding.

\section{Practice of Cloud Computing in SMEs}

\subsection{Evolution Steps of SMEs From IT to Cloud Computing}

Traditional enterprise's IT focuses on the internal management, supports the transactional applications as a tightly coupled system. Web2.0 supports interactive services. It is a loosely coupled system and adds value to the traditional enterprise's IT. The cloud computing focuses on the external markets, to enhance the competitiveness of enterprises in the external market, supports the shared services as a loosely coupled system. The cloud computing is not a new technology but a new service delivery model that changes the traditional IT service. When the IT of SMEs evolves towards the cloud computing, the following steps can be taken: the first step is integration, standardization and optimization of the data center; the second step is to achieve the transformation of data center by virtual technology or other methods; the third step is to build a flexible and dynamic IT infrastructure, and realize the cloud computing. If some SMEs can't build their own cloud computing architecture, they can lease IT resources to achieve 
their business demands. They should take full consideration of the integration with the original IT transaction so as to avoid confusion and audit responsibilities[5].

\subsection{Cloud Computing Architecture of SMEs}

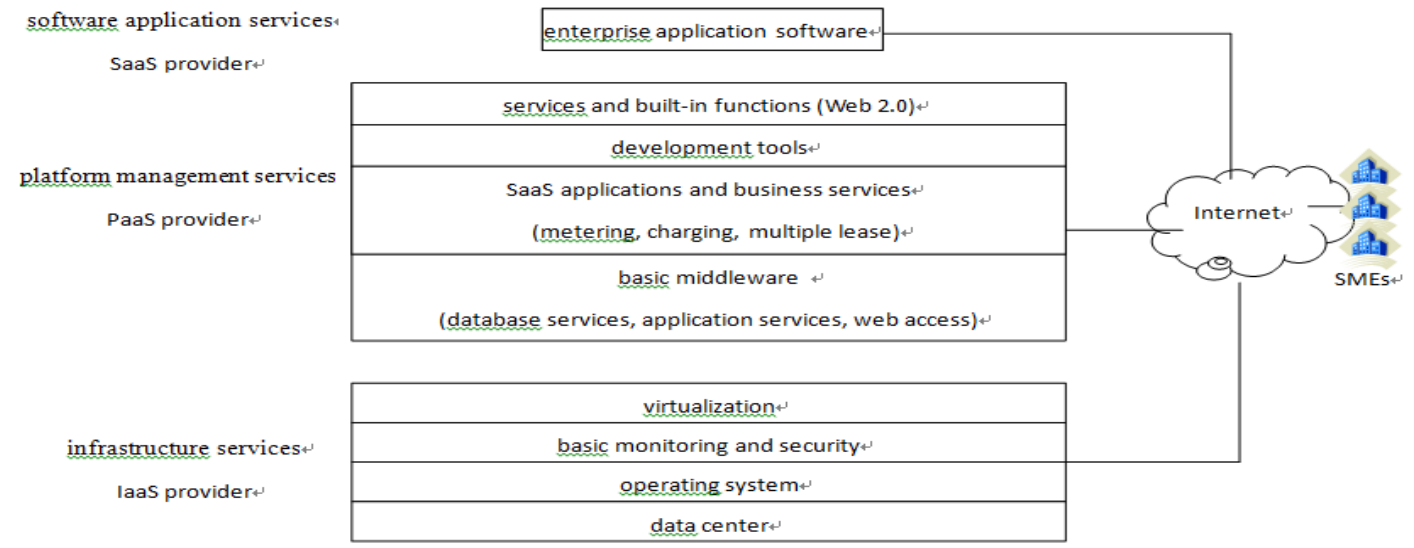

Figure 1: Cloud Computing Architecture of SMEs

The cloud computing architecture of SMEs should include the infrastructure as a service (IaaS), the platform as a service (PaaS), the software as a service (SaaS). The architecture is shown in Fig. 1.

The cloud computing infrastructure is mainly composed of servers, networks and storage devices. For servers, the cloud computing requires it to have more intensive computing power (multi channel and multi core structure), full virtualization capabilities (CPU instruction virtualization, software virtualization, chip virtualization), integration of multiple I/O (data access and storage) and dynamic scheduling of computing resources[8]. For network, it requires 100Gbps network performance, flat and highly scalable architecture, large scale two layer acyclic network for virtual machine environment, unified exchange of FCOE/DCE and L2-L7 deep high speed(10GE performanc)Security integration. For storage, it requests high speed I/O (10Gbps-16Gbps ) [9], virtual mass storage, automated storage management and storage security and FCOE for unified exchange and I/O integration. The cloud computing is changing the IT service model because it'sgreatly improved the performance of IT infrastructure, cloud computing focuses on high performance, virtualization, dynamicity, scalability, flexibility and security. Fig. 2 shows the focuses on architecture of the cloud computing.

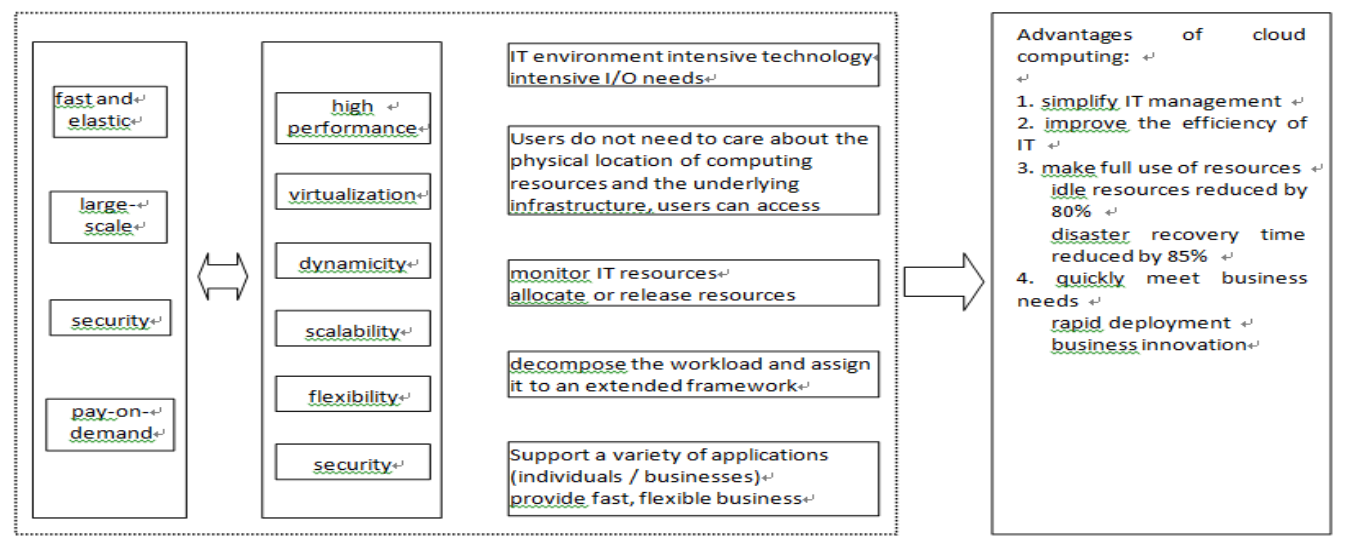

Figure 2: Focuses on Architecture of Cloud Computing 


\subsection{Cloud Computing Model of SMEs}

The capability of operation and maintenance is not so strong due to inadequate infrastructure. SMEs can use the following cloud computing model:

$\mathrm{u}$ Enterprise leasing, operation and maintenance outsourcing and private using of resources

The cloud service providers establish basic resources while being responsible for the management and maintenance of resources and theenterprises lease cloud resources that are provided by cloud service providers, but these physical resources shall be exclusively used by enterprises as a kind of virtual hosting service.

$\mathrm{u}$ Enterprise leasing, operation and maintenance outsourcing, resource sharing

The cloud service providers establish the basic resources while being responsible for the management and maintenance of resources, the enterprises lease cloud resources that are provided by cloud service providers, and multiple companies lease resources provided by the cloud service providers at the same time within the cloud framework. There is a virtual isolation between enterprises. It formats the mode of shared private cloud.

u Public cloud services

The cloud service providers provide Internet oriented public services for SMEs (such as email, instant messaging and disaster recovery, etc.), cloud architecture connect with public network. The cloud service providers are responsible for security of the user's data.

\section{Analysis of Cloud Computing Security in SMEs}

\subsection{SMES' Secure Migration Toward The Cloud}

For the consideration of the performance-price ratio, SMEs may choose more public cloud services, but the risk of public cloud security is higher than the private cloud[7], so SMEs should take the following measures to avoid the risk of migration to public cloud:

u Choose cloud service providers with good reputation and high credibility.

u Migrate to cloud selectively. For these important data or sensitive information, SMEs should do all tests and verification, migrate to cloud cautiously.

u Do the safety management and monitoring after business migration. On the one hand, verify the security measures from cloud service provider by technical means and standard audit; on the other hand, do a good job of their own system security management and monitoring, including key information backup, etc.

u Understand the service agreements, privacy agreements in detail, use agreement terms to safeguard their rights and interests, for example, the cloud service providers need to update the protection system timely, return data, provide backup data and return process monitoring information to SMEs at the end of the contract.

u Know clearly the location that is provided by cloud service providers for storing the enterprise's data, control the location of the data storage as much as possible, and pay attention to avoiding legal disputes arising from legal differences in different countries and regions.

\subsection{Iaas Security Strategy for SMEs}

It includs the security of servers, storage devices, networks, systems and virtual data center. When the operation of the enterprise migrates to the cloud, the company's resources and 
data are placed in the data center of cloud service provider. The physical security of data center is particularly important. Measures: 1. Limit the staff authorized to access data center. The minimum privilege policy shoule be used. Only those who are in need can obtain privileges and manage applications and services. 2. Authentication. In the standard security protocol, for the authentication of site items, access tokens and logs, etc. It is important to identify the required authentication clearly. If high sensitive data needs to be accessed, multi factor authentication is required. 3. Resource access monitoring. The operation and maintenance control mechanism shoule be used for automatic monitorin, early warning shoule be provided in the event of abnormal situations and problems, and audit records should be prepared including the information of application, approval, access and delivery, etc. 4. Deploy firewall, anti-virus wall, intrusion detection and other protective systems.

\subsection{PaaS Security Strategy for SMEs}

PaaS provides the application framework. Thesoftware developers can build new applications on this framework, or extend existing applications, and it frees the SMEs from complex and inefficient job which needs to establish, configure and maintain the program development environment. Measures: 1. Identity and Access Management. Identity authentication/single login can simplify the user experience in application access, reduce the risk and management burden; identity cycle management manages the delivery of the enterprise to ensure the accuracy of enterprises' rights. Companies should also consider the use of union authentication to enhance portability of existing rights between internal systems and cloud services. 2. Information Protection. The data security is used to prevent accidental data leakage, protect privacy and ensure integrity; the protection of intellectual property rights is to protect business and trade secrets as well as information assets; the encryption is used to protect confidential information in storage or transmission; the digital signature is used to protect the integrity of data in the transmission and verify the reliability of the stored information; 3. continuity of service. In the cloud computing model, allowing multiple cloud service providers to participate in safety procedures, including security monitoring, auditing, legal liability, incident response, business continuity, which needs to take care of the demands of all participants.

\subsection{SaaS Security Strategy for SMEs}

Measures 1. Software development security. Any software development organization should follow the process of design and development so that the software has security and function of privacy protection, security and privacy protection should be taken into account on every stage of development, such as requirements, design, implementation, verification, release and response. 2. Software application security. Auditing and logging are used to record the time, the place, the person and the thing; authentication is used to prove identity; suthorization is used to define what permissions a user has and what things can be done; the encryption technology is used to ensure the security of data transmission; the configuration management is used to specify the identity of the application running, which database is connected, and how to manage and how to protect, etc; the algorithm is used to determine the method of protecting the confidentiality and prove the integrity of data; the exception management is used to give a 
method for handling application errors and exceptions; the sensitive data management provides the protection of sensitive data in memory, network and stored procedures; the session management is used to process and protect the interaction between the user and the application; the validation ensures that the input information received by the application is valid and secure, and determines whether the data from the database and shared file are trusted.

\section{Conclusion}

Our research team has established a link with local company in Yunnan Province of China, and provided the practical cloud computing strategies for this company, including the security recommendations. They were effective and successful. It is worth mentioning that, in the practice of cloud computing, some valuable experience and lessons have been gained. They will provide reference for related research in the future.

The practice has shown that the cloud computing can take on a variety of highperformance roles in the rapidly changing business environment, help SMEs to reduce costs, improve management, promote the development of information technology and integrate resources. Therefore, in the fierce business competition environment, the cloud computing is the best strategy and practice to achieve "two integrations" in China. It is the key factor to form and enhance the competitiveness of SMEs.

\section{References}

[1] Li Liping,Wu Xiaosong. Framework and Strategy of Customer Relationship Management System in Electronic-commerce.CeNet[C].2016:341-348.indexed by EI

[2] Lei Wanyun. Cloud Computing Technology, Platform and Application [M]. Beijing: Tsinghua University Press, 2011:88-318

[3] Barney,J.B.Firm Resources and Sustained Competitive Advantage.Journal of Management[J],2012(6):99-120

[4] Gary Garrison and Sanghyun Kim and Robin L.Wakefield.Success Factors for Deploying Cloud Computing.Communications of the ACM,2012(9):23-31

[5] Feeny,D.F. and Willcocks,L.P.Core IS Capabilities for Exploiting Information Technology.Sloan Management Review,2012(3):9-21

[6] Zaheer,A. and Venkatraman,N.Relational.Governance as an Interorganizational Strategy:An Empirical Test of the Role of Trust in Economic Exchange.Strategic Management Journal[J],2013(5):373-392

[7] A.Jukan and D.Katsaros and Y.Goeleven.Architectural Requirements for Cloud Computing Systems:An Enterprise Cloud Approach.J Grid Computing[J],2011(9):3-26

[8] Ma Fei. The Thinking of Current Status of Cloud Computing Security and China's Policy. Telecommunication Network Technology[J], 2015 (2):8-10

[9] Lei Wanyun. Information Construction Strategy of Group Enterprise in Post Crisis Era. E manufacturing Journal[J], 2009(11):22-24 